\title{
Photo-stimulated luminescence and photo-induced infrared absorption in $\mathrm{ZnWO}_{4}$
}

\author{
Minoru Itoh ${ }^{\mathrm{a}, *}$, Tsuyoshi Katagiri ${ }^{\mathrm{a}}$, Tomonori Aoki ${ }^{\mathrm{a}}$, Masami Fujita ${ }^{\mathrm{b}}$ \\ ${ }^{a}$ Department of Electrical and Electronic Engineering, Shinshu University, \\ Nagano 380-8553, Japan \\ b Japan Coast Guard Academy, Kure 737-8512, Japan
}

Reflection, luminescence-excitation, luminescence decay kinetics, photo-stimulated luminescence, and photo-induced infrared absorption have been measured for $\mathrm{ZnWO}_{4}$ crystals in the temperature range of 6-300 K. The results are compared with the previous data. The optical properties of $\mathrm{ZnWO}_{4}$ are discussed on the basis of the present results, as well as the result of the relativistic molecular orbital calculation.

Keywords: scintillation material; $\mathrm{ZnWO}_{4}$; electronic structure; optical property; decay kinetics

\footnotetext{
* Corresponding author: Tel.: +81-26-269-5261; Fax: +81-26-269-5220;

E-mail address: itohlab@shinshu-u.ac.jp (M. Itoh)
} 


\section{Introduction}

Zinc tungstate $\mathrm{ZnWO}_{4}$ crystallizes in the wolframite structure, and has been known to be one of the important scintillating and laser-host materials. Recently, $\mathrm{ZnWO}_{4}$ attracts renewed attention because it is a promising candidate for the search of weakly interacting massive particles (Mikhailik and Kraus, 2006). However, its fundamental optical properties, in particular the electronic band structure, have not been so much investigated as compared with other tungstates such as $\mathrm{PbWO}_{4}$ and $\mathrm{CaWO}_{4}$ with the scheelite structure.

In the present work, we have carried out various types of experiments for $\mathrm{ZnWO}_{4}$ crystals: reflection, luminescence-excitation, luminescence decay kinetics, photo-stimulated luminescence, and photo-induced infrared absorption. The results obtained are compared with those previously reported for this material. Based on the present data, we discuss the optical properties of $\mathrm{ZnWO}_{4}$ by referring to the recent result of the relativistic molecular orbital calculation (Itoh, Fujita, Inabe, 2006).

\section{Experiment}

Single crystals of $\mathrm{ZnWO}_{4}$ were grown by the flux method at the Professor Oishi's laboratory of Shinshu University. The concentration of any unwanted impurities, e.g., Mo or Fe ions, was at a level below $10 \mathrm{ppm}$. A fleshly cleaved crystal with the (010) face was mounted on the sample holder in a closed-cycle He optical cryostat, allowing a temperature variation between 6 and 300 K.

The experiments of reflection and photo-induced infrared (IR) absorption were performed by using synchrotron radiation from the UVSOR storage ring as a light source. For the latter experiment, the specimen was two-photon excited by the 2nd harmonics ( $3.1 \mathrm{eV}$ ) of a regenerative amplified Ti:sapphire laser. The transmission measurements were carried out with a Michelson interferometer operated in rapid scan mode. The photo-induced absorption was obtained from the difference between the transmitted IR intensities with and without the laser excitation.

The luminescence spectra were measured through a $30 \mathrm{~cm}$ monochromator equipped with a photomultiplier or an ICCD camera under the excitation with UV light from a $200 \mathrm{~W} \mathrm{D}_{2}$ lamp. Recombination luminescence was stimulated with red light from a He-Ne laser just after turning off the UV light. The stimulated light signal was recorded as a short-lived spike on a chart 
recorder (response time $\leq 0.3 \mathrm{~s}$ ). The luminescence spectra were corrected for the dispersion and spectral response of the detection system.

The fourth harmonics (4.66 eV) of a Q-switched Nd:YAG laser was employed for excitation of the intrinsic absorption region of $\mathrm{ZnWO}_{4}$. The power density was varied by using a suitable combination of filters. The decay curves of luminescence were detected by a photomultiplier and displayed on a digital storage oscilloscope.

\section{Results}

Fig. 1 shows the reflection spectrum observed at $6 \mathrm{~K}$ under near-normal incidence with the light polarized parallel to the crystalline $c$-axis. The result is similar to that reported by Nagirnyi et al., 2002. One can see a sharp lowest-energy peak at $4.37 \mathrm{eV}$. A broad band with three fine structures is observed in the region between 4.5 and $7.5 \mathrm{eV}$. Two distinct bands appear at around $9 \mathrm{eV}$. Some broad structures are seen in the high-energy region above $12 \mathrm{eV}$.

Fig. 2 shows luminescence spectra measured at several different temperatures under the excitation with $4.66 \mathrm{eV}$ photons. The peak position is located at $2.48 \mathrm{eV}$ at $8 \mathrm{~K}$, and is blue-shifted by $0.03 \mathrm{eV}$ with increasing temperature up to $300 \mathrm{~K}$. The excitation spectrum for the $2.48 \mathrm{eV}$ luminescence is presented by a solid line in Fig. 3, together with the reflection spectrum (dotted line) on an expanded scale. The excitation threshold is located at $3.9 \mathrm{eV}$. These results are fairly consistent with the previous data (Nagirnyi et al., 2002; Kolobanov et al., 2002).

Photo-stimulated luminescence (PSL) spectrum at $8 \mathrm{~K}$ was confirmed to be the same as that shown in Fig. 2. The excitation spectrum of PSL is depicted by open circles in Fig. 3. The PSL appears when $\mathrm{ZnWO}_{4}$ is excited with UV light in the region above $4.9 \pm 0.2 \mathrm{eV}$. The present result in Fig. 3 is in conflict with the data by Nagirnyi et al., 2002, in which the intensity of PSL exhibits a sharp increase at energies exceeding $5.7 \mathrm{eV}$.

The decay curves of the luminescence at $2.5 \mathrm{eV}$ under two different power densities (20 $\mathrm{mW} / \mathrm{cm}^{2}$ and $50 \mu \mathrm{W} / \mathrm{cm}^{2}$ ) of laser light are shown in Fig. 4. When the power density is high, the

decay curves are best fitted by a sum of three components (fast, medium, and slow); $\tau_{\mathrm{f}}=1.0 \pm 0.2$ $\mu \mathrm{s}, \tau_{\mathrm{m}}=11 \pm 1 \mu \mathrm{s}$, and $\tau_{\mathrm{s}}=46 \pm 3 \mu \mathrm{s}$ at $8 \mathrm{~K}$, and $\tau_{\mathrm{f}}=1.0 \pm 0.2 \mu \mathrm{s}, \tau_{\mathrm{m}}=6 \pm 1 \mu \mathrm{s}$, and $\tau_{\mathrm{s}}=24 \pm 2$ $\mu \mathrm{s}$ at $300 \mathrm{~K}$. Under low-density excitation, the decay curve consists of two components of $1.0 \pm$ 0.2 and $50 \pm 3 \mu$ s at $8 \mathrm{~K}$, while it is described by a single exponent with $\tau=24 \pm 2 \mu$ s at $300 \mathrm{~K}$. 
These values are partially in accordance with the previous results (Babin et al., 2004; Chernov et al., 2004; Mikhailik and Kraus, 2006).

Fig. 5 shows the photo-induced IR absorption spectrum at $28 \mathrm{~K}$. A broad band is observed in the region above $7000 \mathrm{~cm}^{-1}$. This IR band decreases in intensity with increasing temperature and disappears above $100 \mathrm{~K}$, as seen from the inset of Fig. 5.

\section{Discussion}

Very recently, the relativistic molecular orbital calculation has been undertaken for $\mathrm{ZnWO}_{4}$ (Itoh, Fujita, Inabe, 2006). The obtained result reveals that the conduction band is built up of the W $5 d$ state, with significant contribution from the $\mathrm{Zn} 4 s$ state in the bottom region. The valence band has largely an $\mathrm{O} 2 p$ character, and the $\mathrm{Zn} 3 d$ state is concentrated in the bottom region of the valence band.

Based on the above calculation, we suppose that, when some perturbation such as the crystal field or local lattice deformation exists, the $\mathrm{Zn} 3 d$ state mixes with the $\mathrm{O} 2 p$ valence state, thus forming the hybridized $\mathrm{Zn} 3 d-\mathrm{O} 2 p$ state in the upper part of the valence band. If this assumption is correct, it is reasonable to assign the lowest reflection peak at $4.37 \mathrm{eV}$ to the excitonic transition in $\mathrm{Zn}^{2+}$ ions. In this connection, it is worth noting that the oscillator strength of the exciton transition in $\mathrm{ZnWO}_{4}$ is significantly small compared with that in $\mathrm{PbWO}_{4}$ (Fujita et al., 2002), where the cationic exciton originates from the $6 s \rightarrow 6 p$ transition in $\mathrm{Pb}^{2+}$ ions.

A large part of the broad band between 4.5 and $7.5 \mathrm{eV}$ in Fig. 1 is ascribed to the interband transition $\mathrm{O} 2 p \rightarrow \mathrm{W} 5 d$. The contribution of the $\mathrm{Zn} 4 s$ state to the $\mathrm{W} 5 d$ state may result in the fine structures appearing on the broad band. Two distinct bands at around $9 \mathrm{eV}$ are likely due to the transitions from the well-isolated $\mathrm{Zn} 3 d$ state to the $\mathrm{Zn} 4 s$ conduction state with an admixture of the $\mathrm{W} 5 d$ state.

The $2.48 \mathrm{eV}$ luminescence is an intrinsic feature of $\mathrm{ZnWO}_{4}$, and is ascribed to the radiative decay of self-trapped excitons (STEs) on the regular $\mathrm{WO}_{6}$ group (Kolobanov et al., 2002). Since this luminescence is excited in the energy region including the lowest exciton band ( $\geq 3.9 \mathrm{eV})$, it is believed that excitons generated optically on $\mathrm{Zn}^{2+}$ ions can move to $\left(\mathrm{WO}_{6}\right)^{6-}$ molecular ions, to emit $2.48 \mathrm{eV}$ photons. In other words, free excitons have a chance to be trapped by lattice defects before their movement to $\left(\mathrm{WO}_{6}\right)^{6-}$. 
The excitation threshold, $4.9 \pm 0.2 \mathrm{eV}$, of PSL corresponds to the band-gap energy $E_{\mathrm{g}}$, above which free electrons and holes are created, and then they radiatively recombine with each other or are trapped by defects or by inducing lattice deformation around themselves. This is consistent with the result that the threshold coincides with the fine structure at $4.95 \mathrm{eV}$ due to the interband transition. The value of $E_{\mathrm{g}}$ for $\mathrm{ZnWO}_{4}$ has been determined to be $4.6 \mathrm{eV}$ from the beginning of the rise of the reflectivity after the excitonic structure (Kolobanov et al., 2002) and $5.7 \mathrm{eV}$ from the excitation spectrum of PSL (Nagirnyi et al., 2002). The present value is close to the former, but is considerably different from the latter. From our result, the binding energy of excitons in $\mathrm{ZnWO}_{4}$ is estimated to be $0.53 \pm 0.2 \mathrm{eV}(=4.9-4.37 \mathrm{eV})$.

It was found that the luminescence decay curves are strongly dependent on the power density of laser light. When the sample is excited with low-power density, the decay curve is really single-exponential at $300 \mathrm{~K}$. On the other hand, the decay curve becomes non-exponential under high-density excitation. A similar power dependence of the decay curve has been found for the STE luminescence in $\mathrm{PbWO}_{4}$ (Itoh et al., 2003). We ascribe the non-exponential decay kinetics to the Auger process of two STEs on adjacent $\left(\mathrm{WO}_{6}\right)^{6-}$ groups, which would result in the non-radiative decay of one STE and the decomposition of another. The coexistence of the fast and slow components at $8 \mathrm{~K}$ under low-density excitation is accounted for in terms of the zero-field-split triplet state of the STE, as in the case of alkali halides (Fischbach et al., 1974).

In the present experiment, a photo-induced IR absorption band was observed above $7000 \mathrm{~cm}^{-1}$ at low temperatures $(<100 \mathrm{~K})$. Koepke and Lempicki (1994) have found a transient absorption band peaking at around $22000 \mathrm{~cm}^{-1}$ in $\mathrm{ZnWO}_{4}$ at $300 \mathrm{~K}$, and ascribed it to the optical transition from the STEs. The photo-induced IR band observed by us is different from the above mentioned band, and would be attributed to some lattice defect produced by the above-gap excitation. Such radiation-induced defects could influence the movement of free carriers, thereby changing the scintillation character of $\mathrm{ZnWO}_{4}$ crystals.

\section{Acknowledgements}

We are deeply grateful to Prof. S. Oishi for supplying the single $\mathrm{ZnWO}_{4}$ crystals used in the present experiments. Our sincere thanks are also due to Mr. H. Nakamura and Dr. J. Azuma for their assistance in the early stage of this work. The present study was partially performed under the Joint Studies Program of the Institute for Molecular Science in Okazaki, and was supported 
by a Grand-in-Aid from the Ministry of Education, Culture, Sports, Science, and Technology of Japan.

\section{References}

Babin, V., et al., 2004. Decay kinetics of the green emission in tungstates and molybdates; Radiat. Meas. 38, 533-537.

Chernov, S., et al., 2004. Luminescence spectra and decay kinetics in $\mathrm{ZnWO}_{4}$ and $\mathrm{CdWO}_{4}$ crystals; Phys. Stat. Sol. B 241, 1945-1948.

Fischbach, J. U., Fröhlich, D., Kabler, M.N., 1973. Recombination luminescence lifetimes and the self-trapped exciton in alkali halides; J. Lumin. 6, 29-43.

Fujita, M., Itoh, M., Horimoto, M., Yokota, H., 2002. Fine structure of the exciton band and anisotropic optical constants in scheelite $\mathrm{PbWO}_{4}$ crystals; Phys. Rev. B 65, 195105.

Itoh, M., Fujita, N., Inabe, Y., 2006. X-ray photoelectron spectroscopy and electronic structures of scheelite- and wolframite-type tungstate crystals; J. Phys. Soc. Jpn. 75, 084705.

Itoh, M., Horimoto, M., Fujita, M., 2003. Luminescence decay of $\mathrm{PbWO}_{4}$ crystals under different excitation conditions; J. Phys.: Condens. Matter 15, 193-201.

Koepke, Cz., Lempicki, A., 1994. Excited-state absorption in $\mathrm{ZnWO}_{4}$ crystal; J. Lumin. 59, 33-37.

Kolobanov, V. N., et al., 2002. Optical and luminescent properties of anisotropic tungstate crystals; Nucl. Instrum. Methods Phys. Res. A 486, 496-503.

Mikhailik, V. B., Kraus, H., 2006. Cryogenic scintillators in searches for extremely rare events; J. Phys. D: Appl. Phys. 39, 1181-1191.

Nagirnyi, V., et al., 2002. Energy transfer in $\mathrm{ZnWO}_{4}$ and $\mathrm{CdWO}_{4}$ scintillators; Nucl. Instrum. Methods Phys. Res. A 486, 395-398.

\section{Figure captions}

Fig. 1. Reflection spectrum of $\mathrm{ZnWO}_{4}$ observed for $E / / c$ in a wide spectral range at $6 \mathrm{~K}$. 
Fig. 2. Luminescence spectra of $\mathrm{ZnWO}_{4}$ excited with $4.66 \mathrm{eV}$ photons at several different temperatures. The intensity at $8 \mathrm{~K}$ is normalized to unity.

Fig. 3. Excitation spectra for the $2.48 \mathrm{eV}$ luminescence (a; solid line) and for the PSL (b; solid line with open circles) measured at $8 \mathrm{~K}$, along with the reflection spectrum (c; dotted line).

Fig. 4. Luminescence decay kinetics at 8 and $300 \mathrm{~K}$ under high- and low-power excitation with laser light at $4.66 \mathrm{eV}$.

Fig. 5. Photo-induced IR absorption spectrum of $\mathrm{ZnWO}_{4}$ at $28 \mathrm{~K}$. The inset shows the temperature dependence of the integrated intensity of the IR absorption band. 


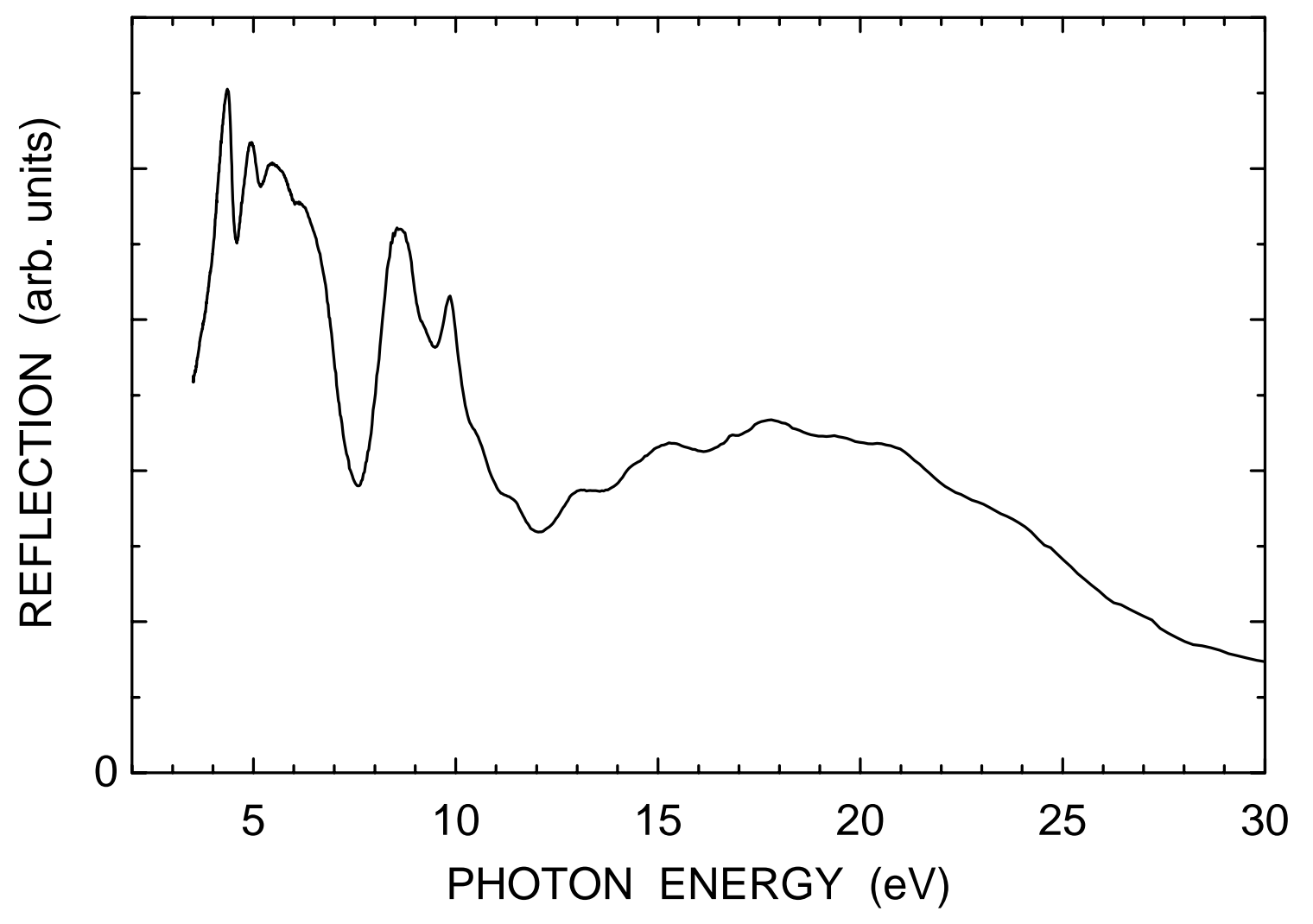

Fig. 1 


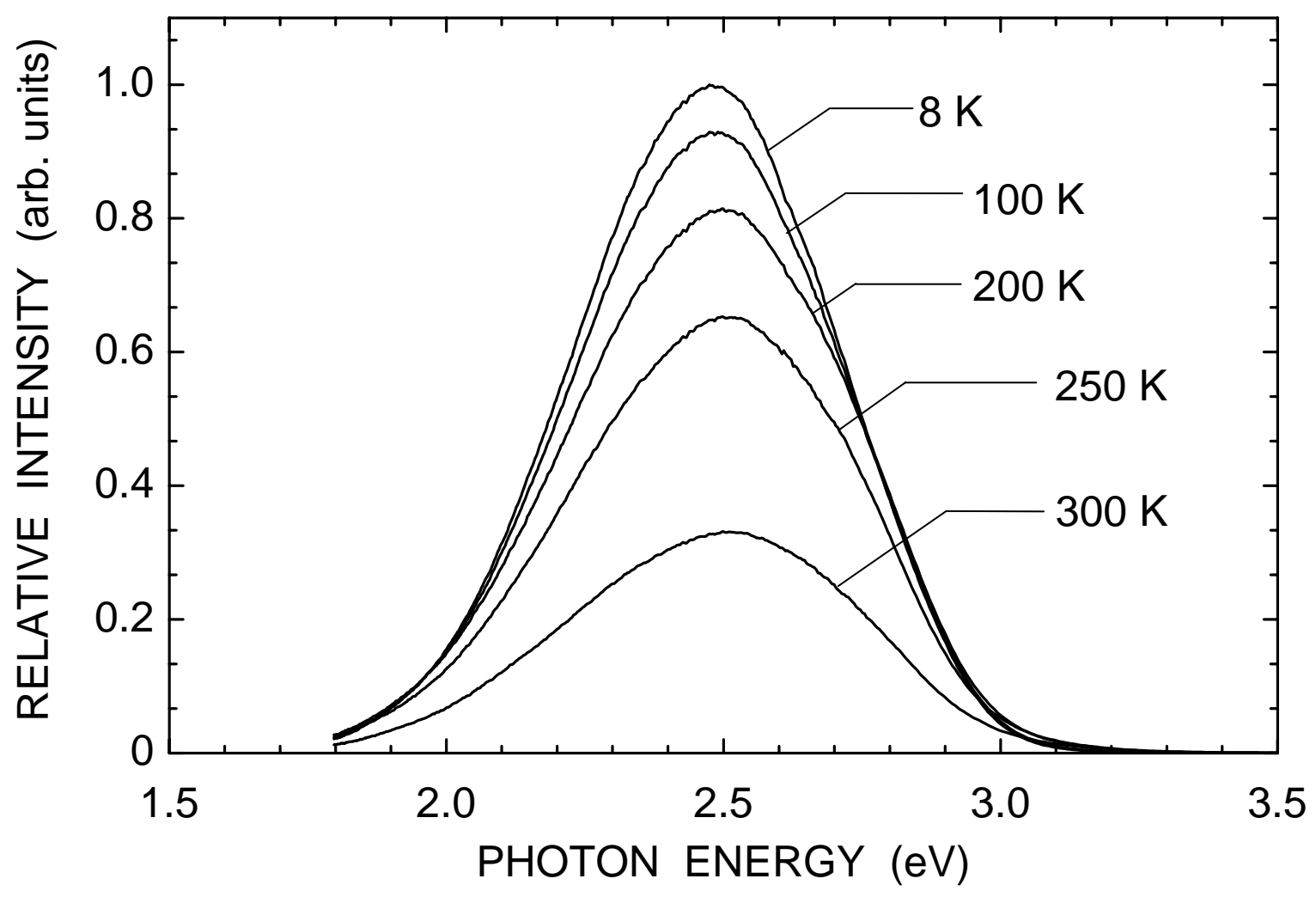

Fig. 2 


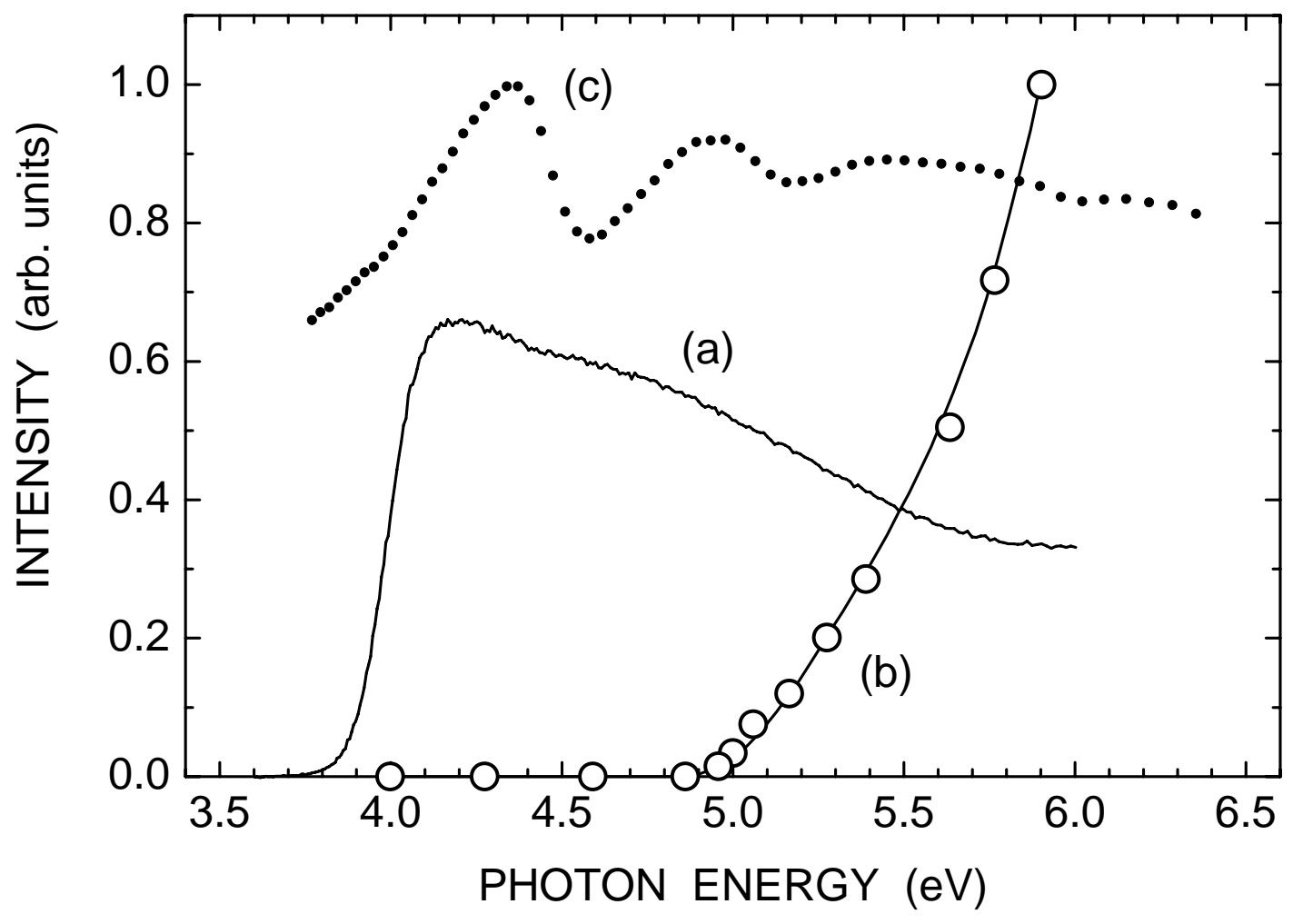

Fig. 3 


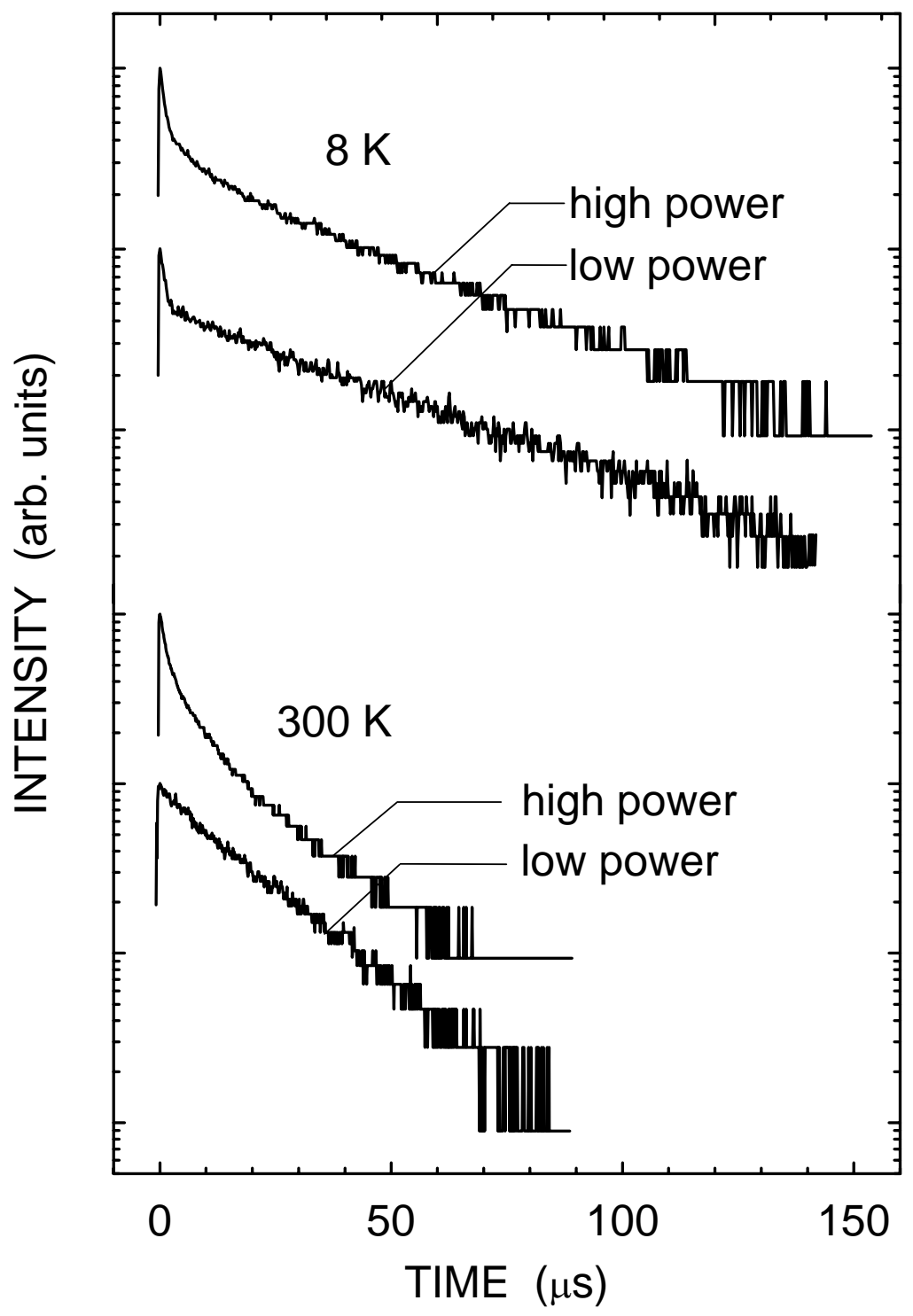

Fig. 4 


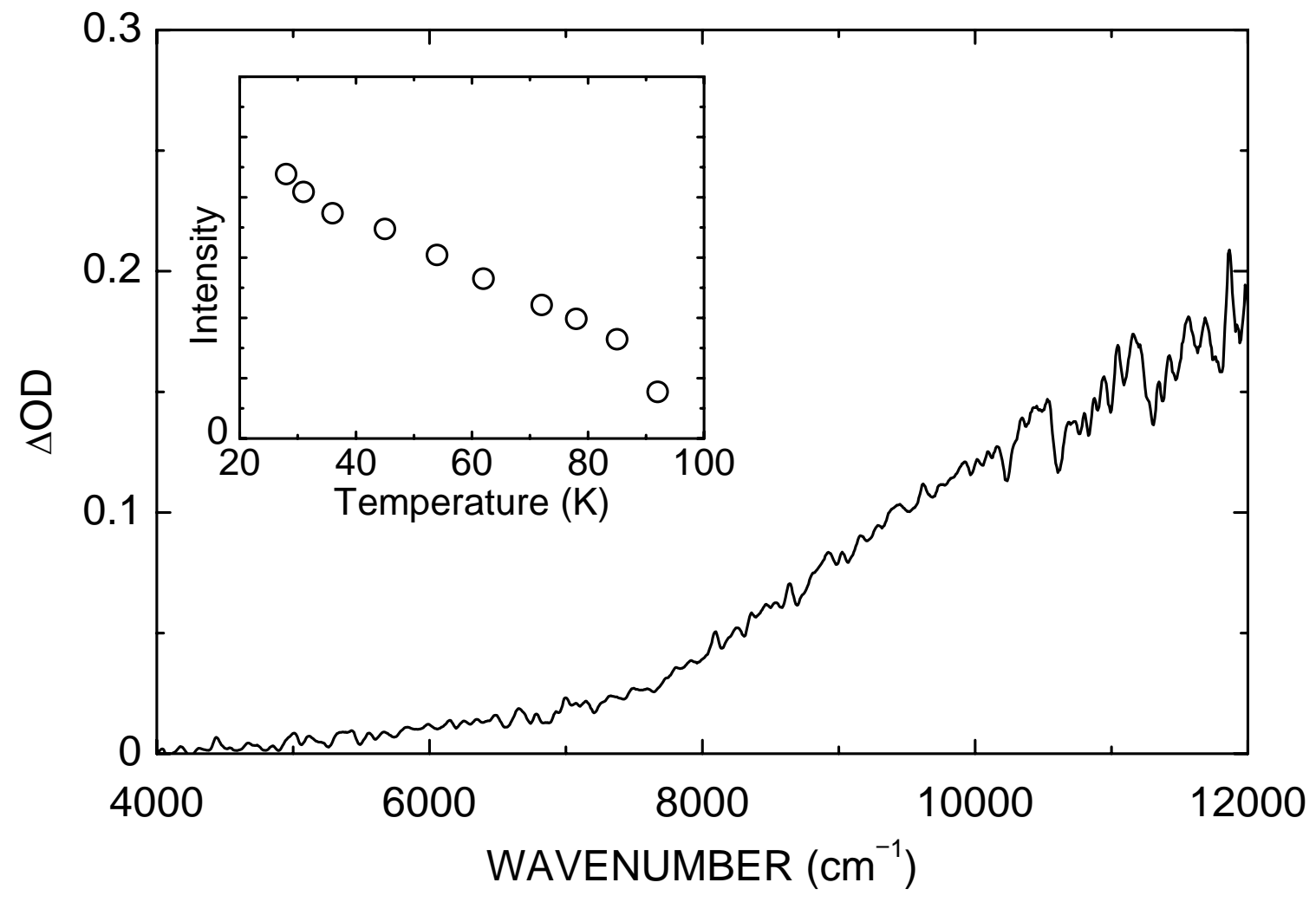

Fig. 5 